\section{Applied Behavior Analysis in Autism Spectrum Disorders in China and Hong Kong}

\section{Abstract}

There is a wealth of evidence detailing the efficacy of Applied Behavior Analysis in the treatment of Autism Spectrum Disorders. However, the existing research knowledge base on the use of this intervention in this population in regions outside of the United States and Europe is far more modest. In particular, there is minimal data on the efficacy of Applied Behavior Analysis in the treatment of individuals with Autism Spectrum Disorders residing in regions of greater China (e.g., the People's Republic of China, Hong Kong). A review of the available literature pertaining to studies of local service programs and supporting policies are presented, accompanied by the discussions of clinical and research implications of applying behavior change strategies among the Autism Spectrum Disorder populations in China and Hong Kong.

Keywords: Autism; Applied Behavior Analysis (ABA); China; Hong Kong; IQ score

Received: July 18, 2017; Accepted: August 01, 2017; Published: August 10, 2017

\section{Kay Chang* and Charles M Zaroff}

\author{
Psychology Department, University of \\ Macau, Macau
}

\begin{abstract}
*Corresponding author: Kay Chang
\end{abstract}

\section{kchang@umac.mo}

Assistant Professor, Psychology Department, University of Macau, Macau,

Tel: +85388228385

Citation: Chang K, Zaroff CM (2017) Applied Behavior Analysis in Autism Spectrum Disorders in China and Hong Kong. Acta Psychopathol. Vol. 3 No. 5: 52.

\section{Introduction}

Autism Spectrum Disorder (ASD) is a neurodevelopmental disorder characterized by persistent deficits in social communication and social interaction, and restricted, repetitive patterns of behavior [1]. While there is no known cure for ASD, interventions are available, interventions that may be particularly beneficial when applied with adequate frequency and intensity. Lovaas seminal study on the use of Applied Behavior Analysis (ABA) in the treatment of ASD revealed a substantial ceiling for children with the disorder [2]. Recent research has further shown that improved social communication and social interaction, and even in some cases, increases in IQ score are possible, when behavioral interventions are applied early enough in development [3].

At present, empirical research clearly supports the use of $A B A$ and other interventions with a behavioral foundation in the treatment of ASD [4]. These results have helped lay the foundation for treatment recommendations prescribed by professional and governmental organizations. Thus, in their treatment prescriptions, the National Institute of Neurological Disorders and Stroke emphasizes the successful outcomes found in many children with ASD who undergo behavioral intervention early in development, and further delineate the components of such intervention as structure and skill-oriented training sessions. In their recommendations, $A B A$ is referred to specifically. In contrast, non-ABA treatments are mentioned only in reference to ancillary symptoms in the individual with ASD (e.g., medication for anxiety), or in recommendations for family members (e.g., counseling).

Nonetheless, in spite of a broad knowledge base detailing the efficacy of $A B A$ in ASD, barriers to its use still do exist. While time and resources required for individualized $A B A$ [5], particularly in children with significant degrees of disorder symptomatology, may be considerable, these are not the only limiting factors. Other barriers to behavioral treatment may reflect the degree of knowledge of ASD. That is, while ASD is considered to have a neurobiological/genetic etiology (reflected in the use of the term neurodevelopmental disorder in the latest revision of the American Psychiatric Association's Diagnostic and Statistical Manual of Mental Disorders [1], confusion about ASD characteristics and etiologies persists in the lay population [6]. Such confusion impacts treatment choice because greater awareness of ASD in the general population has been found to correlate with a younger age at diagnosis [7]. In turn, a younger age at diagnosis contributes to trends towards earlier intervention, which is itself associated with better developmental outcomes [8].

\section{Knowledge of ASD}

Knowledge of ASD may also interact with geographic region of residence. For instance, in community samples in Western cultures, misconceptions about ASD etiology (e.g., conceptualizations based on psychodynamic conceptualizations of 'cold' parenting) are generally in the minority. However, in 
other regions, such as the People's Republic of China (referred to herein as China), misconceptions pertaining to etiology persist [9], and associated findings suggest that the prevalence of 'autism spectrum conditions' is potentially under-diagnosed and under-detected (e.g., 26.6 per 10,000 across China, Taiwan and Hong Kong [10]). With such results in mind, research into the use of empirically validated, behavioral-based interventions for ASD in this region is needed.

\section{In order to examine trends in the use of such behaviorally-oriented treatments for ASD in China and Hong Kong, the current report investigates the results of an English-language literature review}

A search on PubMed revealed 115 citations for 'behavior autism China,' and 31 citations for 'behavior autism Hong Kong' (in contrast to 3,071 such citations when 'United States' was substituted for 'China'). When the search term was narrowed to 'applied behavior analysis autism,' 60 such citations were found for the United States, but only five such citations were found when 'China' was used as a search term. Most citations from the aforementioned searches did not directly address research reports on behavioral interventions for ASD in these regions. Thus, the terms 'China' and, 'Hong Kong' were also entered into internal journal search engines for the following journals devoted to behavioral treatments: Analysis of Verbal Behavior, Behavior and Philosophy, Behavior and Social Issues, Behavior Modification, Behavioral Interventions, Behavioral Technology Today, Education and Treatment of Children, European Journal of Behavior Analysis, Journal of Applied Behavior Analysis, Journal of Behavior Therapy, Journal of Behavioral Education, Journal of Positive Behavior Interventions, Journal of the Experimental Analysis of Behavior, The Behavior Analyst Today.

All published studies using these search terms were reviewed. Additionally, in order to enable a more comprehensive analysis, reports cited within papers originally captured under the initial search terms were also reviewed for inclusion eligibility. Inclusion criteria included research took place in either China or Hong Kong, with individuals with an ASD diagnosis, with interventions with a behavioral basis (including, but not limited to, ABA). In total, only six studies specifically utilized behavioral interventions for ASD as part of an empirical report. The lack of a larger body of evidence prevents the use of statistical procedures like meta-analysis, or the use of procedures employed as part of a systematic review. Thus, the review supports the significant gap in overall research efforts of the efficiency nature of the ABA application for the autistic population in the China and Hong Kong region.

A note of interest, by and large the research published in China and Hong Kong regions tends to focus on sensory integration approaches. More specifically, interventions studies have often included (cultural specific) treatments such as needle stimulation, traditional Chinese mind-body exercises, and electro-acupuncture [11]. Regardless, there is an absence of evidence that such an approach is contraindicated in China and Kong. In a 'Cultural Considerations' section of the report by Wang [12], parents were described as receptive and eager to learn the behavioral strategies imparted to them, despite the western orientation and origination of the intervention (although the possibility that program components were spontaneously modified by parents was noted).

While overall, little attention has been paid to the manner in which behavioral intervention approaches may require modification for cultural acceptability; this topic has not gone completely ignored. Lau et al. found that parent training programs based on behavioral principles with Chinese immigrant families in a non-clinical/nonASD sample were efficacious. Their suggestions for behavioral intervention modifications for use within the Chinese culture consisted simply of slowing the pace at which skill lessons are conducted, and increasing the quantity of behavioral rehearsal.

Wang et al. [13] in a report discussing positive behavior support practices, provide an in depth look at value differences across cultures, and how these may be best approached in order to implement needed behavioral supports. Among these differences are those manifested in the nature of the partnerships involved (e.g., directness and assertiveness versus maintenance of harmony), those concerning functional assessments of behavior (a focus on the future versus that of the past), and those related to the definition of meaningful life outcomes (e.g., individualism versus promotion of the family unit). While their prescription for addressing (if not directly reconciling) these differences is directed towards practitioners in the United States, these prescriptions may hold weight for practitioners regardless of ethnicity whose training has oriented them to a more western-culture paradigm perspective. They first emphasize the importance of self-awareness of one's own cultural values in conjunction with attempts to gain knowledge of other cultures. Clearly, there is room for behavioral-based intervention and $A B A$ in the treatment of ASD in China and Hong Kong. In a recent commentary, Leaf et al. [14] argue that $A B A$, being a science, is progressive, and thus allows for flexibility. While not addressed explicitly in their commentary, it is suggested herein that this flexibility would allow for the use of ABA across cultures, enabling its fit into individual cultures, while still adhering to the central tenets of the intervention (e.g., empiricism, data collection).

\section{Obstacles on ABA and ASD}

It should be noted that many of the obstacles, barriers, and stresses inherent in research into the application of behaviorally-based interventions in general, and $A B A$ in particular, to individuals with ASD, are in no way, shape, or form specific to China or Hong Kong. Concerning research in the social sciences and beyond, there may be a real implicit bias against replication studies. Recent trends in the ability to register some studies beforehand with specific peer reviewed academic publications may lessen this bias. This trend may have specific implications for attempts at replication across cultures. A rhetorical question posed by Wang [12] bears repeating when addressing this issue: 'why spend time and limited resources in 'reinventing the wheel' when the best practices and evidencebased parent training curricular are available from the developed countries...' They pose a succinct yet important response: with more research initiated by indigenous researchers in the area of intervention, more evidence will become available concerning culturally specific adaptations and modifications needed for successful outcomes. 
Of note, a review of research reports should in no way be interpreted to suggest an absence of an ABA approach in clinical or academic contexts, in schools and centers in China and Hong Kong. This is because reports detailing the actual use of intervention methods for ASD in China and Hong Kong based on behavioral principles, and ABA in particular, are as common, if not more prevalent, than those reports summarized above which report on research in this area. Challenges notwithstanding, ABA appears to be a valued technique in China and Hong Kong, from the perspective of parents of children with ASD, and from professionals involved in the care and education of this group. With improved awareness and knowledge of ASD in China, parents of children with the disorder have increasingly recognized the importance of early intervention, and behavioral therapy [15]. This has led to a rapid and continued increase in the number of parents seeking such services. As a result, some research has focused on how this desire and need is being addressed.

ABA is generally offered at private centers for ASD in China. Zhou et al. [16], in what is likely the most comprehensive account of such centers, estimated their number at greater than 1,000. In their survey of 100 member centers of the Heart Alliance, an industry association of ASD intervention centers created by the Beijing Stars and Rain Education Institute for Autism (the preeminent center for ASD treatment in China), they found a total of 5,360 children being treated, of whom, 3,957 had received a clinical diagnosis of ASD. In 99 of these centers, ABA intervention was offered (as were sensory integration intervention in 73 of the centers, and TEACCH in 36). In these 100 centers, 73 worked with both children and their parents, 24 worked with the child only, and three worked only with parents.

In Hong Kong, as of a 2010 report by Mak and Kwok [17], approximately 30 non-governmental organizations were in existence providing supportive services to children with ASD, one (Heep Hong Society) specifically providing services oriented to individuals with ASD using a TEACCH program for children two to six years of age. Additionally, specialized university-based supports are also available [18]. However, the overwhelming majority of students with ASD in Hong Kong are educated in public schools. Only one privately funded school for ASD in Hong Kong exists, the Autism Partnership School, which provides individualized services using principles of $A B A$, with intensive instruction for staff and multiple levels of staff $A B A$ expertise. Of note is that the center provides services only under the $A B A$ rubric, and thus, the authors of a report on the center specifically mention services not provided, such as those pertaining to sensory integration.

Thus, there are specialized institutes for individuals with ASD in both China and Hong Kong, and behavioral intervention, and ABA in particular, seem to be the treatment of choice. Nonetheless, challenges remain. While these centers tend to possess some quality control procedures for providers [19] training may be problematic. In China, the empirical criteria behind the length of training in some such centers lacks an empirical rationale [19], and there are as of yet no standards for determining who exactly can provide services such as ABA in China [20]. The lack of staff training has been suggested as one of the reasons why many children with ASD in China and Hong Kong fail to make adequate progress, or exhibit significant improvements (even after receiving behavioral intervention) [21].

Perhaps most notable are issues pertaining to the availability of such services. Wong and Kwan [22] make reference to waiting lists for services in Hong Kong that may be as many as three years in duration. Costs too, are a significant concern. In China, ASD training centers tend to be run as primary schools which require payment, the majority of which must come from parents [19]. This may be among the most crucial obstacles, as Wang et al. [15] found that care for children with ASD exceeded annual total household income in nearly one-fifth of the urban households surveyed, and this ratio was even higher in rural households (1/3). Of note, behavioral therapy accounted for the largest proportion of healthcare expenses, with more than $50 \%$ of expenses directed in this manner, doubling the cost of transportation, and tripling the cost of outpatient care for these children. Similarly, costs at the Autism Partnership School in Hong Kong are over 28,000 USD annually.

Unfortunately, outside of such centers, little in the way of treatment is available. Sun et al. [23] note that in China there exists no specific intervention programs or special assistant programs in mainstream schools for children with ASD, and Huang and Wheeler [24] suggest that the typical class size in China (e.g., approximately 50 to 75 students) almost certainly puts a limit to the amount of more individualized attention and services available for a student with ASD.

On a more upbeat note, Wang et al. [15] point out that since 2006, The China Disabled Person's Federation has begun rehabilitation support programs for ASD for low income families. Thus, each family of a child with ASD may receive about 10,000 RMB annually (approximately 1,500 USD), and some municipalities may further supplement this income. They do describe the financial support from governmental agencies for children with ASD as being in a 'very early stage,' and further note that while The China Disabled Person's Federation (at the time of their report) had planned to create early intervention settings for children with ASD in 31 cities in China, few such cities were providing financial support for the families needing these services. Nonetheless, the recognition of the need for such services by governmental agencies is encouraging. Similar trends have been observed in Hong Kong as well. The Department of Education began the Integrated Education Pilot Project towards improving the availability of opportunities and social interactions of students with (and without) disabilities in mainstream schools in 1997. As a result, schools admitting students with special needs receive extra funding towards equipment, alterations for accommodation, and staff support [25], resulting in an increase of students with special needs in mainstream schools.

Of note, obstacles to behavioral intervention for ASD may also arise from parents. In China, parents may be reluctant to accept a diagnosis of ASD [23], and, in perhaps a related finding, only $20 \%$ reported that they would contact a specialist in ASD if needing help (most reported a greater probability of seeking out of a psychotherapist [9]). Interestingly, from a parent perspective, McCabe [20] found a great emphasis on the quantity of intervention approaches relative to their quality. In 
China, parents tend to look for the latest 'equipment' at centers, and a trend was detected in which all of the centers surveyed by McCabe highlighted their ability to provide instruction using all of the 'most advanced methods,' including ABA, TEACCH, Relationship Development Intervention, and sensory integration. One professional interviewed by the author suggested that parents might think that ABA is 'useless,' and a treatment whose 'time has passed, because it was an earlier method, now it's old, and maybe older things don't have much value.' A theme related to this was a tendency for parents to seek different treatments if one was not working, or not working quickly enough.

These concerns on the part of parents and providers speak to concerns that transcend cultural boundaries. Comprehensive $A B A$ continues to be one of only two interventions classified as well-established for ASD [26]. Even its alternates are among the few approaches defined as 'probably' (e.g., individual, focused $A B A$ for augmentative and alternative communication) or 'possibly' (comprehensive ABA classrooms) efficacious. However, any intervention in ASD is often inevitably concerned with designing an intervention for a child with comorbid impairments, and treatment is often intensive and complex. Thus, availability of well trained professionals is always a crucial issue, and professional training issues are not limited to China and Hong Kong. A descriptive survey of university programs (on-campus) pre-approved by a nonprofit agency identifying credentialing needs of individuals undergoing training towards providing $A B A$ (Behavior Analyst Certification Board ${ }^{\circledR}$, Inc.; BACB) identified no more than four such programs in any country outside of the United States (including one in China).

This survey highlights the need for greater advocacy in expediting the creation of such programs outside of the United States. Of greater concern is that even in regions within the United States, in which BACB-approved programs flourish, questions concerning who implements and coordinates behavior-based services may persist [27]. Furthermore, for children and adolescents with ASD who do not receive comprehensive and individualized ABA, education generally occurs in inclusive classrooms which often lack skill coverage for the effective teaching of students with the disorder, and this is the case even in special education classrooms [27]. And of course, cost is always an issue. Echoing what is found in China and Hong Kong; families in the United States are described as being in 'desperate' need of financial support in order to access services [28].

An interesting side note to this discussion concerns the ways in which China and Hong Kong, relative to the United States, might be better equipped going forward to provide services for individuals with ASD. In the report describing the Autism Partnership School in Hong Kong, the authors specifically mention the school's beneficial staff-student ratios and intensive staff training, approaches that might potentially be constrained in a budget-driven society such as the United States. They also discuss challenges in the United States owing to the inability to terminate poorly performing teachers (which occurs in the Autism Partnership School) resulting from a unionized staff.

\section{Summary and Conclusion}

In summary, there are few reports utilizing behavioral-based interventions more broadly, and Applied Behavior Analysis specifically, in China and Hong Kong, for Autism Spectrum Disorders. Nonetheless, what reports do exist, methodological limitations aside, exhibit evidence of efficacy? Limitations in the clinical use of this approach in China and Hong Kong are based in part on culture-specific barriers, but also obstacles more universal in nature. Of the available studies, the positive indications of the application of $A B S$ are encouraging and shall inform an increasingly comprehensive and systematic investigation. The current report thus argues for more awareness and contribution to the study and treatment of ASD on the basis of behavioral principles in China and Hong Kong.

\section{References}

1 American Psychiatric Association (2013) Diagnostic and statistical manual of mental disorders ( $5^{\text {th }}$ edn.). Arlington, VA: American Psychiatric Association.

2 Lovaas OI, Koegel R, Simmons JQ, Stevens Long J (1973) Some generalization and follow-up measures on autistic children in behavior therapy. J Appl Behav Anal 6: 131-165.

3 Eikeseth S, Smith T, Jahr E, Eldevik S (2007) Outcome for children with autism who began intensive behavioral treatment between ages 4 and 7: a comparison controlled study. Behav Modif 31: 264-278.

4 Remington B, Hastings RP, Kovshoff $H$, Degli Espinosa F, Jahr E, et al. (2007) Early intensive behavioral intervention: outcomes for children with autism and their parents after two years. Am J Ment Retard 112: 418-438.

5 Carr EG, Granpeesheh D (2008) Applied behavior analysis: the big picture and ultimate goals. Autism Adv 4: 8-10.

6 Al Anbar NN, Dardennes RM, Prado-Netto A, Kaye K, Contejean Y (2010) Treatment choices in autism spectrum disorder: the role of parental illness perceptions. Res Dev Disabil 31: 817-828.

7 Fernell E, Gillberg C (2010) Autism spectrum disorder diagnoses in Stockholm pre-schoolers. Res Dev Disabil 31: 680-685

8 Fenske EC, Zalenski S, Krantz PJ, McClannahan LE (1985) Age at intervention and treatment outcome for autistic children in a comprehensive intervention program. Anal Interv Devl Disabil 5: 49-58.

9 Wang J, Zhou X, Xia W, Sun CH, Wu L, et al. (2012) Autism awareness and attitudes towards treatment in caregivers of children aged 3-6 years in Harbin, China. Soc Psychiatry Psychiatr Epidemiol 47: 1301-1308.

10 Sun X, Allison C, Matthews FE, Sharp SJ, Auyeung B, et al. (2013) Prevalence of autism in mainland China, Hong Kong and Taiwan: a systematic review and meta-analysis. Mol Autism 4: 7.

11 Chen WX, Wu-Li L, Wong VCN (2008) Electroacupuncture for children with autism spectrum disorder: pilot study of two cases. J Altern Compl Med 14: 1057-1065.

12 Wang P (2008) Effects of a parent training program on the interactive skills of parents of children with autism in China. J Policy Pract Intellect Disabil 5: 96-104.

13 Wang BM, McCart A, Turnbull AP (2007) Implementing positive behavior support with Chinese American families: Enhancing cultural competence. J Posit Behav Interv 9: 38-51. 
14 Leaf JB, Leaf R, McEachin J, Taubman M, Ala'i-Rosales S, et al. (2016) Applied behavior analysis is a science and, therefore, progressive. J Autism Dev Disord 46: 720-731.

15 Wang J, Zhou X, Xia W, Sun CH, Wu LJ, et al. (2012) Parent-reported health care expenditures associated with autism spectrum disorders in Heilongjiang province, China. BMC Health Serv Res 12: 7.

16 Zhou WZ, Ye AY, Sun ZK, Tian HH, Pu TZ, et al. (2014) Statistical analysis of twenty years (1993 to 2012) of data from mainland China's first intervention center for children with autism spectrum disorder. Mol Autism 5: 52.

17 Mak WWS, Kwok YTY (2010) Internalization of stigma for parents of children with autism spectrum disorder in Hong Kong. Soc Sci Med 70: 2045-2051.

18 Wong VC, Hui SLH (2008) Brief report: emerging services for children with autism spectrum disorders in Hong Kong (1960-2004). J Autism Dev Disord 38: 383-389.

19 Sun X, Allison C, Auyeung B, Matthews FE, Murray S, et al. (2013) Service provision for autism in mainland China: a service providers' perspective. Res Dev Disabil 34: 440-451.

20 McCabe $H$ (2013) Bamboo shoots after the rain: development and challenges of autism intervention in China. Autism 17: 510-526.
21 Huang AX, Jia MX, Wheeler JJ (2013) Children with autism in the people's Republic of China: Diagnosis, legal issues, and educational services. J Autism Dev Disord 43: 1991-2001.

22 Wong VC, Kwan QK (2010) Randomized controlled trial for early intervention for autism: a pilot study of the autism 1-2-3 project. $J$ Autism Dev Disord 40: 677-688.

23 Sun X, Allison C, Auyeung B, Baron-Cohen S, Brayne C (2013) A review of healthcare service and education provision of Autism Spectrum Condition in mainland China. Res Dev Disabil 34: 469-479.

24 Huang AX, Wheeler JJ (2007) Including children with autism in general education in China. Childhood Educ 83: 356-360.

25 https://www.policyaddress.gov.hk/pa98/english/erpt/eembedu.pdf

26 Smith T, ladarola S (2015) Evidence base update for autism spectrum disorder. J Clin Child Adolesc Psychol 44: 897-922.

27 Croen LA, Najjar DV, Ray GT, Lotspeich L, Bernal P (2006) A comparison of health care utilization and costs of children with and without autism spectrum disorders in a large group-model health plan. Pediatrics 118: E1203-E1211.

28 Maurice C, Mannion K, Letso S, Perry L (2001) Parent voices: difficulty in accessing behavioral intervention for autism; working toward solutions. Behavioral Interv 16: 147-165. 